\title{
Isotropic “QuasiFluid” Metamaterials Designed by Topology Optimization
}

\author{
Zhang, Huikai; Kang, Zhan; Wang, Yiqiang; Wu, Wenjun
}

Published in:

Advanced Theory and Simulations

Link to article, DOI:

10.1002/adts.201900182

Publication date:

2020

Document Version

Peer reviewed version

Link back to DTU Orbit

Citation (APA):

Zhang, H., Kang, Z., Wang, Y., \& Wu, W. (2020). Isotropic "QuasiFluid" Metamaterials Designed by Topology Optimization. Advanced Theory and Simulations, 3(1), [1900182]. https://doi.org/10.1002/adts.201900182

\section{General rights}

Copyright and moral rights for the publications made accessible in the public portal are retained by the authors and/or other copyright owners and it is a condition of accessing publications that users recognise and abide by the legal requirements associated with these rights.

- Users may download and print one copy of any publication from the public portal for the purpose of private study or research.

- You may not further distribute the material or use it for any profit-making activity or commercial gain

- You may freely distribute the URL identifying the publication in the public portal

If you believe that this document breaches copyright please contact us providing details, and we will remove access to the work immediately and investigate your claim. 


\title{
Isotropic 'quasi-fluid' metamaterials designed by topology optimization
}

\author{
Huikai Zhang ${ }^{1}$, Zhan Kang ${ }^{1 *}$, Yiqiang Wang ${ }^{2}$, Wenjun $\mathrm{Wu}^{1}$ \\ ${ }^{1}$ State key Laboratory of Structural Analysis for Industrial Equipment, Dalian \\ University of Technology, Dalian 116024, China \\ ${ }^{2}$ Department of Mechanical Engineering, Solid Mechanics, Technical University of \\ Denmark, Nils Koppels Alle, B. 404, DK 2800, Lyngby, Denmark \\ *Corresponding author. E-mail address: zhankang@dlut.edu.cn
}

\begin{abstract}
Bi-mode artificial metamaterials have anisotropic mechanical properties, with the ratio of bulk modulus and shear modulus approaching an infinite value in ideal conditions. As a type of 'quasi-fluid' material composed of solid material, it has potential applications in the design of functional devices with desired acoustic, thermal, and mechanical performance. However, the microstructures of such metamaterials are currently mostly determined by parameter synthesis on the basis of existing heuristic configuration designs, which may considerably restrict their topologies and shapes. In this study, new octagon and hexagonal honeycomb bi-mode metamaterials (two-dimensional) are designed with a more systematic approach based on the independent point-wise interpolation method of topology optimization. The objective function is defined as a weighted combination of the bulk and shear moduli. By tuning the values of different weighted coefficients, the transition mechanism can be acquired from the regular microstructure to the bi-mode metamaterial with needle-like or double-cone rods. It is also found that simply increasing the volume
\end{abstract}


fraction in the single material design cannot further improve the target performance, but introducing a small amount of hard material into the design domain can noticeably enhance the bulk modulus. One representative optimized microstructure is fabricated by $3 \mathrm{D}$ printing with stainless steel and polymer materials. Uniaxial quasi-static compression tests and finite element simulations reveal the layer-wise deformation modes of the bi-mode 'quasi-fluid' metamaterial and its capacity to absorb external energy.

\section{Introduction}

Artificial metamaterials can exhibit remarkable physical properties that extend far beyond the properties of their constituent materials. ${ }^{[1]}$ Milton and Cherkaev ${ }^{[2]}$ initially demonstrated the possibility of achieving specified elasticity tensors by combining soft and hard materials. They designed bi-mode (for two-dimensional cases) and penta-mode (for three-dimensional cases) metamaterials. Such metamaterials have anisotropic mechanical properties with high bulk modulus, but low shear modulus. It is noted that strict-sense bi-mode or penta-mode metamaterials with perfect hinges (with zero rotational stiffness) are not mechanically stable under shear strains, and therefore cannot be used in real engineering.

Kadic et al. ${ }^{[3]}$ fabricated the penta-mode and bi-mode metamaterials proposed by Milton and Cherkaev ${ }^{[2]}$ using polymer 3D printing. In contrast to the 'perfect' hinges in the idealized conceptual design with an infinite figure of merit $\left(\alpha_{\mathrm{FOM}}\right.$, which is the 
ratio between the bulk and shear modulus), the diameters $d$ of the connecting hinges were set to be finite values. Also, the $\alpha_{\text {FOM }}$ values become larger as the diameter of the hinges decreases, resulting in a 'quasi-fluid' microstructure. Some cross-media assemblies of solids and fluids with a large ratio $B / G$ can also be regarded quasi-fluid materials. ${ }^{[4]}$

With different anisotropic stiffness and shear properties, the bi-mode (or penta-mode) metamaterial designs given by Milton and Cherkaev ${ }^{[2]}$ have been extended to various applications. For instance, Layman ${ }^{[5]}$ presented a bi-mode design formed by tuning parameterized oblique honeycomb lattice, which can be tailored for acoustic applications. The penta-mode metamaterial was also used to implement an elasto-mechanical unfeelability cloak. ${ }^{[6]}$ Following this, two-dimensional bi-mode metamaterial designs for mechanical cloaks based on direct lattice transformation were investigated. ${ }^{[7,8]}$ In these works, some graded bi-mode metamaterials were generated by tuning the basic parameters of the double-cone configuration. In addition, such metamaterials have also been used to design water-like cloaks, ${ }^{[9]}$ seismic base-isolation devices, ${ }^{[10,11]}$ wave filters ${ }^{[12]}$ and phononic crystals. ${ }^{[13]}$ Recently, research works on the mechanical responses of metallic penta-mode metamaterials with high compression modulus and energy absorbing capacity have also attracted increasing interest. ${ }^{[14,15]}$ Double-cone rhombic dodecahedron lattices with plastic joints have also been reported, which can improve the compression modulus and initial yield strength. ${ }^{[16]}$ Generally, most bi-mode or penta-mode metamaterial designs are based on the configurations described by Milton and Cherkaev, ${ }^{[2]}$ which to some 
extent restricts the design space.

Topology optimization techniques, including the method of solid isotropic material with penalization (SIMP),$^{[17]}$ the level set method ${ }^{[18]}$ and the bi-directional evolutionary structural optimization method, ${ }^{[19]}$ have been used in the design of mechanical metamaterials, ${ }^{[20-23]}$ bionic microstructures, ${ }^{[24-27]}$ optical metasurfaces, ${ }^{[28,29]}$ photonic structure $^{[30,31]}$ and additive manufactured products. ${ }^{[32]}$ Sigmund $^{[33]}$ designed bi-mode and penta-mode metamaterials in the form of discretized truss or frame structures. Mendez et al. ${ }^{[34]}$ and Podesta et al. ${ }^{[35]}$ optimized acoustic cloaking devices composed of a needle-like orthogonal anisotropic honeycomb-like bi-mode metamaterial using inverse homogenization. However, systematic methods for designing bi-mode metamaterials still need to be exploited.

In this work, a topology optimization model is proposed to design bi-mode metamaterials. Specifically, the independent point density interpolation (iPDI) ${ }^{[36}$ model is adopted to represent the topologies of the microstructures to be optimized. The optimized bi-mode microstructures, which have double-cone shaped bar members, possess relatively high bulk modulus but low shear modulus. Mechanical properties, including the elastic modulus and Poisson's ratio, of the new optimized octagon and bi-material bi-mode microstructures are investigated. For studying the load bearing capacity and failure modes of the optimized bi-mode metamaterials, 3D printed metallic and polymer specimens are tested through quasi-static compression experiments. 


\section{Results}

Theory and method. For two-dimensional staggered composite microstructures composed of a soft material (red color) and a reinforcing hard material (blue color) (Inset I in Figure 1a), there are three orthogonal deformation modes, namely tension in the lateral direction, tension in the vertical direction, and shear deformation (Insets II-IV in Figure1a). The effective elasticity tensor $£$ of the microstructure can be computed by the asymptotic homogenization method (AHM) (see Supporting Information). It can be written in matrix form as $[£]_{3}=[\mathbf{\Phi}]^{\top} \lambda[\boldsymbol{\Phi}]$, where $\left.[\lambda]=\operatorname{diag}\left\{\lambda_{1}, \lambda_{2}, \lambda_{3}\right\}, \quad[\Phi]=\int^{1} \Phi^{1}\right\},\left\{\Phi^{2}\right\},\left\{\Phi^{3}\right\}_{\mathrm{H}}^{\mathrm{u}}, \quad \lambda_{i} \quad$ and $\quad\{\Phi\}^{i} \quad(i=1,2,3)$ are respectively the three eigenvalues and orthogonalized eigenvectors of the elasticity matrix. A two-dimensional bi-mode metamaterial is characterized by the property that its effective elastic tensor $£$ has a large eigenvalue related to a hard deformation mode, so that the material cannot deform easily under the corresponding external loads (Inset II in Figure1a). It also has another two small eigenvalues corresponding to the two easy deformation modes, so that it cannot resist certain loading conditions (Insets III and IV in Figure 1a). Besides, a bi-mode metamaterial has an anisotropic mechanical property with a high bulk modulus but a low (or nearly zero in the ideal case) shear modulus.

For an isotropic material, the bulk modulus and shear modulus can be expressed as $B=\left(\sum_{i, j}^{2} £_{i i j j}\right) / 4$ and $G=£_{1212}$, respectively. ${ }^{[37]}$ The mechanical property of an anisotropic bi-mode metamaterial can be characterized by the Figure of Merit (FOM) 
$\alpha_{\mathrm{FOM}}=B / G \cdot{ }^{[3,6]}$

In the optimization formulation, it is particularly important to define a suitable objective function to generate reasonable designs. Maximizing the bulk modulus and minimizing the shear modulus are generally two conflicting design objectives. On the basis of the previous discussion, we see that $\alpha_{\mathrm{FOM}}$ is to be maximized in the bi-mode metamaterial design. In order to avoid instability of the optimization process, we state the optimization problem as a multi-objective optimization model to maximize the objective function $f(\tilde{\mathbf{\rho}})=(1-w) B-w G$. Here, $0 \leq w \leq 1$ is the weight factor and $\tilde{\boldsymbol{\rho}}$ is the vector of design variables in the iPDI method (see Supporting Information). Note that the design problem degenerates into a single-objective optimization problem for maximization of the bulk modulus when $w=0$, or minimization of the shear modulus when $w=1$. With a suitable weight factor $w$, this objective function is able to generate transitional designs with desired properties ranging from extremely high bulk modulus to extremely low shear modulus. 

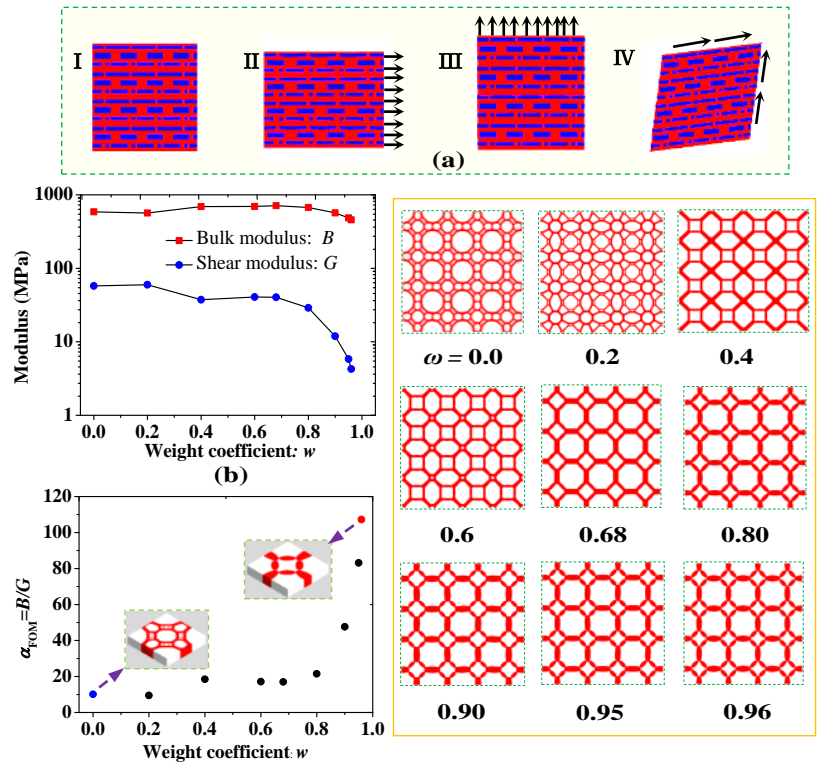

(c)

(d)

Figure 1. Schematic illustration of a bi-mode metamaterial and optimized designs. a) Inset I is a staggered composite material (red and blue colors represent the soft material and the reinforcing hard material, respectively); Insets II and III are the deformed configurations under tension in lateral and vertical directions, respectively; Inset IV is the configuration under shear deformation. b), c) and d) are the optimization results of a two-phase microstructure, where b) presents the curves of the bulk and shear moduli v.s. the weight factor $w, \mathrm{c}$ ) presents the FOM value $\alpha_{\text {FOM }}$, and d) presents nine periodic arrays of optimized microstructures obtained for different values of the weight coefficient.

The material volume constraint and the isotropy constraint are also imposed in the optimization model. The design variables are updated by the method of moving asymptotes (MMA) using the design sensitivities of the objective and constraint functions.

Details of the optimization formulation and sensitivity analysis scheme are provided in Supporting Information. 
Design optimization results. We first consider a square design domain with a side length $a=90 \mathrm{~mm}$ (see Figure S4a and the initial material density layouts shown in Figure S4c, in Supporting Information). Young modulus and Poisson's ratio of the basic solid material are $E=10 \mathrm{GPa}$ and $v=0.3$, respectively.

For the volume fraction $V=0.3$ and different values of the weight factor $w$, the optimized bulk and shear moduli are given in Figures $1 \mathrm{~b}$, and the corresponding values of $\alpha_{\text {FOM }}$ are given in Figure 1c (see Table S1 in Supporting Information). The optimized periodic microstructures obtained with different values of the weight coefficient are also given in Figure 1d. It is seen that the shear modulus decreases gradually as the weight factor increases in the range of $w<0.8$, but drops dramatically when $w>0.8$. The optimized microstructures (denoted by the red point) located at the right-upper region in Figure 1c are characterized by some double-cone structures linked by weak joints. Such microstructures result in significant reduction of the shear modulus. Specifically, when the weight factor $w=0.96$, the optimized design achieves the highest FOM value of $\alpha_{\mathrm{FOM}}=107.2$. Here, the low shear modulus is mainly attributed to the microstructural configuration formed by the double-cone bars. However, the microstructures obtained with this optimization model have very small effective stiffness (e.g. bulk modulus), as seen in Figure 1b. This hinders further increase of the FOM value.

In order to obtain desired bi-mode property while still ensuring sufficient stiffness of the microstructure, we impose a lower bound constraint of $0.1 \mathrm{MPa}$ on the
Slettet:

Formateret: Fremhæuning 
effective shear modulus of the microstructure in the optimization model. For the weight factor $w=0.8$, the iteration history of the FOM value is shown in Figure 2a (the corresponding volume fraction and the objective function value are given in Figure S6). Here, the maximum iteration steps is specified to be 200. From the figures, it is seen that the objective function exhibits a steady increase (Figure S6) and finally achieves the optimal FOM value of $\alpha_{\mathrm{FOM}}=1326.2$. The bulk and shear moduli are $B=132.6 \mathrm{MPa}$ and $G=0.1 \mathrm{MPa}$, respectively. The final optimized microstructure (Figure 2a) is also composed of double-cone bar members. The eigenvalues of the effective elastic matrix $[\square]$ (in Figure 2a) are $\lambda_{1}=265.24, \lambda_{2}=0.2$, and $\lambda_{3}=0.1$, and the corresponding orthogonal eigenvectors are $\left\{\Phi^{1}\right\}=[1 / \sqrt{2}, 1 / \sqrt{2}, 0]^{\mathrm{T}}$, $\left\{\Phi^{2}\right\}=[1 / \sqrt{2},-1 / \sqrt{2}, 0]^{\mathrm{T}}$ and $\left\{\Phi^{3}\right\}=[0,0,1.0]^{\mathrm{T}}$. Here, $\lambda_{2}$ and $\lambda_{3}$ are three orders of magnitude smaller than $\lambda_{1}$, which indicates that the optimized microstructure can be regarded a two-dimensional bi-mode metamaterial.

For the optimized bi-mode metamaterial microstructure, the two eigenvectors $\left\{\Phi^{2}\right\}$ and $\left\{\Phi^{3}\right\}$ are the easy deformation modes, and $\left\{\Phi^{1}\right\}$ is the hard deformation mode. We apply the strain fields $\left\{\varepsilon^{k}\right\}=\left\{\Phi^{k}\right\}(k=1,2,3)$ to such a bi-mode microstructure, and then compute the corresponding stress fields using the generalized Hooke's law $\left\{\sigma^{k}\right\}=[£]\left\{\varepsilon^{k}\right\} \quad(k=1,2,3)$, where $\left\{\sigma^{k}\right\}$ is the vector of stress induced by the applied strain $\left\{\varepsilon^{k}\right\}$. The predicted stress fields are $\left\{\sigma^{1}\right\}=[187.55,187.55,0]^{\mathrm{T}} \mathrm{MPa},\left\{\sigma^{2}\right\}=[-0.141,0.141,0]^{\mathrm{T}} \mathrm{MPa}$, and $\left\{\sigma^{3}\right\}=[0,0,1]^{\mathrm{T}} \mathrm{MPa}$. 
purpose of numerical verification of the optimized microstructure, a $10 \mathrm{~mm} \times 10 \mathrm{~mm}$ geometrical model of the optimized unit cell was reconstructed through the commercial software platform Abaqus (Inset I in Figure S7 a). Bi-axial and shear displacement loads related to the three strain fields $\left\{\varepsilon^{k}\right\}(k=1,2,3)$ were applied on the boundaries of the bi-mode unit cell, as shown in Figure S7. We found that the deformation mode $\left\{\varepsilon^{1}\right\}$ had a strain energy of $16897.9 \mathrm{~J}$, while the deformation modes $\left\{\varepsilon^{2}\right\}$ and $\left\{\varepsilon^{3}\right\}$ yielded much smaller strain energy values of $0.0039 \mathrm{~J}$ and $0.003 \mathrm{~J}$, respectively. Besides, the effective Poisson's ratio is often of interest in mechanical metamaterial design. For an isotropic two-dimenaional metamaterial, the Poisson's ratio can vary between -1 to $1 .^{[33]}$ In our pevious work, using the homogenization method, we showed that an extreme Poissons ratio $v=-1$ had been achieved through the negative Poissons ratio metamaterial design. ${ }^{[21]}$ With the same homogenization procedures, the Poisson's ratio of the optimized isotropic bi-mode metamaterial obtained in this study is predicted to be 0.998 , which is close to the theoretical upper bound 1 .

We then verified the effective Poisson's ratio of the optimized metamaterial with a tessellated finite element model. A $7 \times 7$ periodic array of the optimized microstructure was constructed and analyzed with Abaqus, where a uniform lateral displacement of $10 \mathrm{~mm}$ was applied to the right edge under the sliding boundary conditions (Figure S7 b). The simulation results show that the average vertical displacements of the top edge is $-9.28 \mathrm{~mm}$, and thus the Poisson's ratio is estimated to be $v=-(-9.28 \mathrm{~mm}) / 10(\mathrm{~mm}) \approx 0.93$. The small discrepancy between this value and 
that predicted by the homogenization method may be attributed by the accuracy of the reconstructed CAD model and the finite size of the periodic array model.

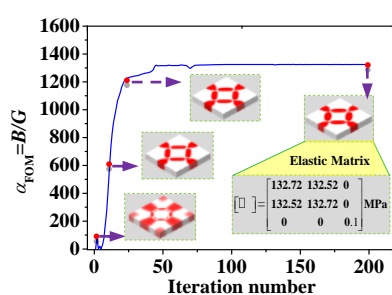

(a)

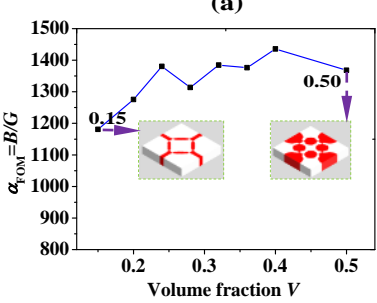

(b)

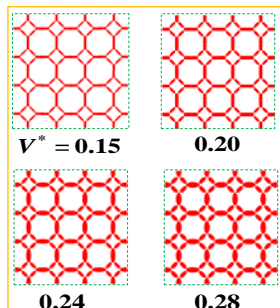

0.28

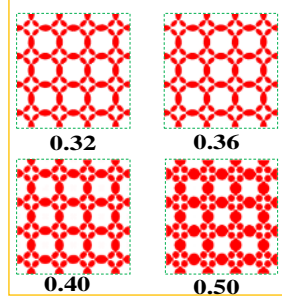

(c)

Figure 2. Bi-mode metamaterial with high values of FOM. a) Iteration histories of the optimization process; b) the FOM values of optimized microstructures obtained for different material volume fractions, and c) optimized periodic microstructure obtained for different material volume fractions.

Influences of volume fraction. Generally, achievable mechanical properties of microstructures strongly depend on the material volume fraction. Figure $2 b$ shows the optimized bi-mode microstructures obtained with different material volume fractions. We find that all of these designs consist of eight double-cone members, and possess values of $\alpha_{\text {FOM }}$ greater than 1000 (in Figure 2b). It is found that the value $\alpha_{\text {FOM }}$ of the optimized microstructure increases with the material volume fraction for the range of $V<0.24$, but barely varies for the range $V>0.24$. When comparing the bi-mode 
metamaterial designs obtained under the low volume fraction $V=0.15$ and the high volume fraction $V=0.32$, one can find that more material is distributed at the middle sections of the double-cone bars rather than at the joints (in Figure 2c), which cannot significantly improve the bulk modulus of the microstructures and their FOM values. Similar conclusions, regarding the elastic modulus of three-dimensional penta-mode metamaterials fabricated by the power bed fusion technique, were also given by Hedayati et al. ${ }^{[14]}$ This finding suggests the limitation of single-material design of bi-model metamaterials in achieving desirable effective stiffness.

Bi-material topological design. In order to further improve the FOM value $\alpha_{\mathrm{FOM}}$ and the stiffness $B$ of the bi-mode microstructures, in the presence of small-sized joints (reducing the effective stiffness $B$ ), we introduced a small amount of harder material into the design domain as a reinforcing phase (denoted in blue color in Figure 1a). Here, the reinforcing material has an relatively high elastic modulus $E_{\mathrm{H}}=20 \mathrm{GPa}$ and a Poisson's ratio $v_{\mathrm{H}}=0.3$. The volume fraction ratios of the hard and soft materials are 0.05 and 0.25 , respectively. The modified multi-material interpolation model (Eq. S7 in Supporting Information) was used in the topology optimization model. The distribution of the two material phases in the optimized multi-material bi-mode microstructures is shown in Figure 3a. It is seen that the microstructure has an overall topology similar to the design shown in Figures 2, and the soft material mainly forms the middle part of the double-cone bars. However, the joints of all the double-cone bars consist of only the reinforcing hard material. From the effective elastic matrix $[\square]$ (see Figure S8 in Supporting Information), it is predicted that this multi-phase 
bi-mode metamaterial has a bulk modulus $B=195.84 \mathrm{MPa}$ and $\alpha_{\mathrm{FOM}}=1958.4$, which are 1.48 times that of the single-phase counterpart (Figure 2a).

The above results show that the topological design and material selection of the joints have a significant effect on the effective mechanical properties of the bi-mode microstructures. In practice, these joints are usually fabricated with a finite small size, and they should have sufficient stiffness to ensure a desired load bearing capacity. To this end, replacing the materials of joints with a harder material becomes a feasible way to increase the FOM value of the bi-mode metamaterial.

Rhombic shaped single-material microstructures have been used by some researchers in mechanical cloaking device design. ${ }^{[5]}$ Therefore, the single-material and multi-material rhombic bi-mode hexagonal microstructures (in Figures $3 b$ and c), obtained with the proposed optimization model in a rhombic design domain_(Figure

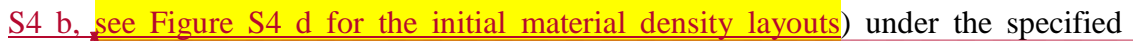
volume fraction $V=0.3$, are also compared in Table S3 of Supporting Information. It is noted that these optimized designs have similar topologies as the microstructures used in acoustic cloaking device designs. ${ }^{[5]}$ The FOM values of the single-material and bi-material designs are $\alpha_{\mathrm{FOM}}=883.8$ and $\alpha_{\mathrm{FOM}}=1070.0$, respectively. Clearly, these rhombic shaped microstructures have relative low bulk moduli as compared with the octagonal microstructures with the same material volume shown in Figure 2a. This comparison shows that the proposed topology optimization model is able to generate bi-mode microstructures with improved stiffness as compared with heuristic or trial-and-error design approaches, while still satisfying the isotropic constraint.

Slettet:

Slettet: Figure S4 b

Formateret: Fremhæuning 


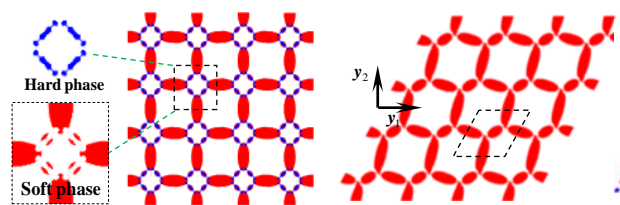

(a)

(b)

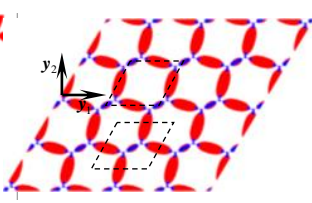

(c)

Figure 3. Comparison of single-material and bi-material designs. a) The hard material, soft material phase and the periodic array of the composite bi-mode metamaterial; b) and c) Rhombic hexagonal honeycomb bi-mode metamaterials composed of single material and bi-material, respectively.

Geometrical model and metallic 3D printing. The small-sized hinges of the optimized bi-mode metamaterial are difficult to be fabricated with 3D printing, therefore a simplified geometrical model with the volume fraction $V=0.3$ was reconstructed (Figure 4a) according to the optimized design shown in Figure 2a. It is composed of $10 \times 10$ periodic unit cells and has a dimension of $40 \mathrm{~mm} \times 40 \mathrm{~mm} \times 8 \mathrm{~mm}$ (Figure $4 \mathrm{~b})$. Each unit cell consists of 12 equicrural triangles $\triangle A B C$, with the angle $\theta^{*}=69.58^{\circ}$ (see Figure S5 for details of evaluation of the approximate isotropy in Supporting Information). Two specimens (Figures 4c) of this model were fabricated with 316 stainless steel via Selective Laser Melting (SLM, EOSINT-M280, for details see Fabrications). The printed specimens and the scanning results by the scanning electron microscopy (SEM, details see Fabrications) are shown in Figures 4c, e and f. Due to the excessive metallic particles attached to the surfaces of the printed specimens, the total masses of printed specimens are larger than their designed values (see Fabrications). Compared with the ideal geometrical model (Figure 4b), these specimens also have some size deviations and surface 
morphology defects (see insets (g)-(i) in Figure 4).

(a)
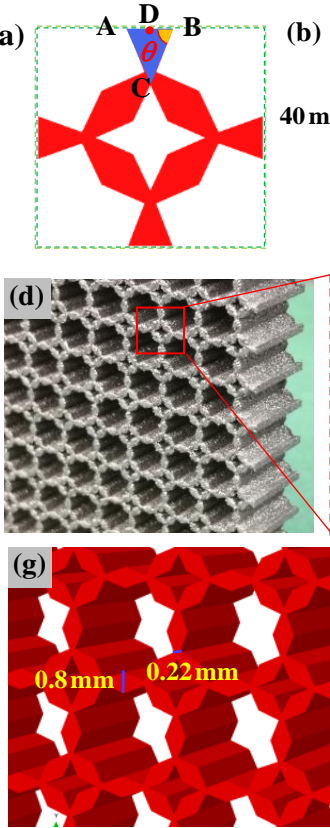

(b)
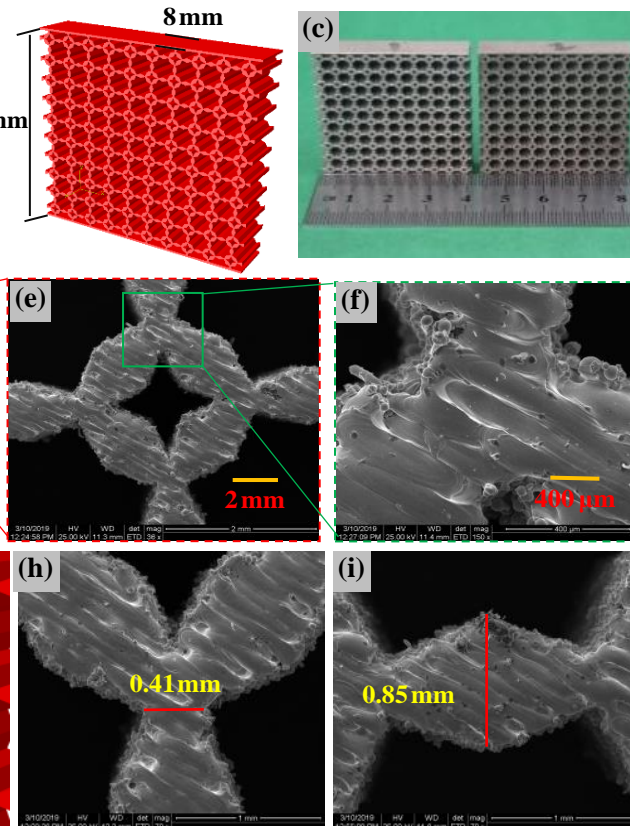
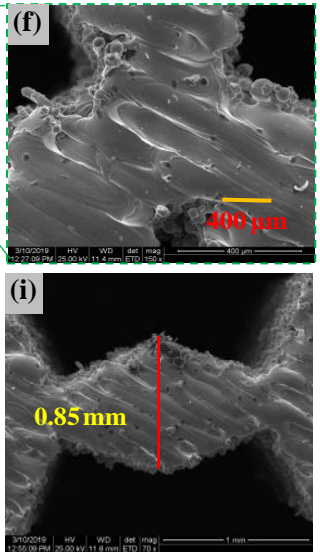

Figure 4. Specimens manufactured by SLM. a) Parametric models of bi-mode microstructure; b) CAD bi-mode periodic structure composed of $10 \times 10$ microstructures with size of $40 \mathrm{~mm} \times 40 \mathrm{~mm} \times 8 \mathrm{~mm}$; c) Two metallic samples (316 stainless steel); d) Zoomed figure of specimen 2; e) and f) SEM scanning results with $\times 36$ and $\times 150$ magnification; g) The local hinge sizes of the ideal CAD model as designed; h) and i) measurements of manufacturing deviations, and the SEM scanning results with $\times 70$ magnification of the $3 \mathrm{D}$ printed specimen.

Compression experiments. Uniaxial compression tests of the two metallic bi-mode metamaterial samples were conducted at room temperature on the MTS New810 testing machine, as shown in Figure 5a. The displacement load was applied to samples 1 and 2 (Figure $5 \mathrm{~b}$ ) at the speed of $1 \mathrm{~mm} / \mathrm{min}$, and the measured load-displacement curves are given in Figure $5 \mathrm{~b}$. We found that the initial buckling occurred on the 
bottom and top regions at the displacement of $2.122 \mathrm{~mm}$. The initial peak load for both samples are $14.5 \mathrm{kN}$. Later, the whole periodic structures could still resist the compression loads with relative stable and high plateau regions. This is partly due to the fact that the stainless steel material has a ductile property. More importantly, the whole structure collapsed in a layer-wise manner (see Figure 5b), because the uniaxial loads also induced shear deformations (the bi-mode metamaterial can barely sustain shear forces). Such a layer-wise deformation mode introduced large hinge rotations and extensive plastic deformations of the unit cells, which improves the energy absorbing capacity of the bi-mode metamaterial.

Such phenomenon is also confirmed through our explicit dynamic finite element (FE) simulations carried out with the software Abaqus. Here, the ideal elastic-plastic model was employed (see Numerical simulation). We find that the simulation results agree with the experiments (see Figure 5b). Some deviations between the experimental and simulation results may be caused by the manufacturing errors of the specimens (see insets (g)-(i) in Figure 4).

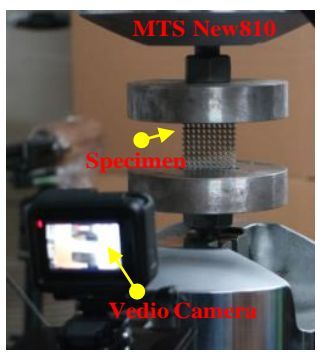

(a)

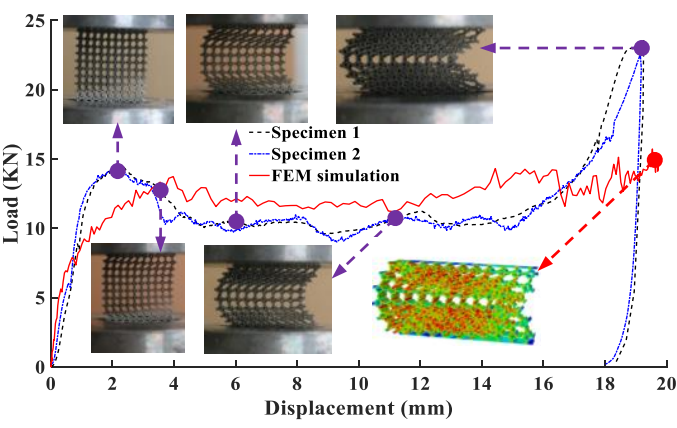

(b) 
Figure 5. Compression test experiments. a) Experiment equipment, including Gopro 7 video camera, MTS New810 tension and compression testing machine; b) Load-displacement curves of the two metallic specimens.

\section{Conclusions and remarks}

In this study, we proposed a topology optimization method for two-dimensional bi-mode metamaterials. The design objective was to maximize the bulk modulus and minimize the shear modulus. New octagon honeycomb microstructural designs with extreme FOM values were obtained with the proposed model. We have also found that the double-cone joints are the dominant factors to determine the bulk modulus and the FOM values. We further came up with bi-material bi-mode metamaterial designs by introducing a hard reinforcing material phase into the design domain to enhance the stiffness. These findings may provide useful guidance for designing new bi-mode functional devices.

A representative parametric bi-mode microstructure was fabricated by $3 \mathrm{D}$ printing with stainless steel metallic and polymer materials. A layer-wise failure mode of the metallic metamaterial samples was observed through the axial compression experiment tests and finite simulations.

In summary, the proposed design method is able to generate bi-mode microstructures with 'quasi-fluid' properties, which can be further exploited to design new function devices such as acoustic cloaks and wave filters. Additionally, It is envisaged that our method can be extended to design a new type of three-dimensional 
penta-mode metamaterials.

\section{Fabrications}

The metallic specimens were fabricated with 316 stainless steel with the Young's modulus $185 \mathrm{GPa}$, yield strength $530 \mathrm{MPa}$, mass density $7.92 \mathrm{~g} / \mathrm{cm}^{3}$ and Poisson's Formateret: Fremhævning Formateret: Fremhæuning ratio 0.4 . The Lase power, scanning speed and layer thickness were $400 \mathrm{~W}, 6 \mathrm{~m} / \mathrm{s}$ and $20 \mu \mathrm{m}$, respectively. The actual masses of the two specimens are $37.2 \mathrm{~g}$ and $37.94 \mathrm{~g}$ (the mass of the ideal CAD model is $32.28 \mathrm{~g}$ ). The specimens were cooled at room temperature for releasing the residual stress.

The metallic microstructure morphologies and geometry deviations were captured through scanning electron microscopy (SEM, FEI Quanta 200). The voltage was $25 \mathrm{KV}$, and the magnification factors were set to be $\times 36$ for the whole unit cell and $\times 150$ for local regions.

The deformations and failure modes were recorded by a video camera (Gopro-7, 6 million pixels)

\section{Numerical Simulation}

A three-dimensional shell model (with thickness $8 \mathrm{~mm}$ ) was constructed for the finite element simulation of the stainless steel specimens. The whole periodic structure was discretized into 15,273 bilinear quadrilateral elements with reduced integration (S4R) and 1,032 bilinear triangular elements (S3). The general contact 
model, with a friction coefficient of 0.4 , was considered in the analysis. The explicit dynamic step was set to be $0.01 \mathrm{~s}$.

\section{Supporting Information}

Supporting Information is available from the Wiley Online Library or from the authors.

\section{Acknowledgements}

Financial support of the National Science Fund for Distinguished Young Scholars (Grant No. 11425207) and the Key Program of National Science Foundation of China (Grant No. U1508209) is gratefully acknowledged. We are also grateful to professor Krister Svanberg from Kungliga Tekniska Hogskolan for proving the optimization package MMA. Special thank also goes to Dr. Ping Wang from Dalian University of Technology for the helpful guidance of experiment tests.

\section{Conflict of Interest}

The authors declare no conflict of interests.

Keywords: Bi-mode; two-dimensional metamaterial; quasi-fluid; topology optimization; layer-wise collapse. 


\section{References}

[1] X. L. Yu, J. Zhou, H. Y. Liang, Z. Y. Jiang, L. L. Wu, Progress in Materials Science, 2018, 94. [2] G. W. Milton, A. V. Cherkaev, Journal of Engineering Materials and Technology-Transactions of the Asme, 1995, 117, 4.

[3] M. Kadic, T. Buckmann, N. Stenger, M. Thiel, M. Wegener, Applied Physics Letters, 2012, 101,4 .

[4] W. Yang, H.T. Wang, T. F. Li, S. X. Qu, Science China-Physics Mechanics \& Astronomy, 2019, $62,1$.

[5] C. N. Layman, C. J. Naify, T. P. Martin, D. C. Calvo, G. J. Orris, Physical Review Letters, 2013, 111, 2 .

[6] T. Buckmann, M. Thiel, M. Kadic, R. Schittny, and M. Wegener, Nature Communications, 2014, 5 .

[7] L. Y. Hai, Q. Zhao, and Y. G. Meng, Advanced Functional Materials, 2018, 28, 30.

[8] T. Buckmann, M. Kadic, R. Schittny, M. Wegener, Proceedings of the National Academy of Sciences of the United States of America, 2015, 112, 16.

[9] A. G. Zhao, Z. G. Zhao, X. D. Zhang, X. Cai, L. Wang, T. Wu, H. Chen, Applied Physics Letters, 2017, 110, 1.

[10] F. Fabbrocino, A. Amendola, G. Benzoni, and F. Fraternali, Ingegneria Sismica, 2016, 33, 1.

[11] A. Amendola, G. Carpentieri, L. Feo, F. Fraternali, Composite Structures, 2016, 157.

[12] Y. Huang, X. G. Lu, G. Y. Liang, Z. Xu, Journal of Applied Physics, 2017, 121, 12.

[13] A. O. Krushynska, P. Galich, F. Bosia, N.M. Pugno, S. Rudykh, Applied Physics Letters, 2018, 113, 20.

[14] R. Hedayati, A. M. Leeflang, A. A. Zadpoor, Applied Physics Letters, 2017, 110, 9.

[15] A. Amendola, C.J. Smith, R. Goodall, F. Auricchio, L. Feo, G. Benzoni, F. Fraternali, Composite Structures, 2016, 142.

[16] X. F. Cao, S. Y. Duan, J. Liang, W.B. Wen, D.N. Fang, International Journal of Mechanical Sciences, 2018, 145.

[17] M. P. Bendsoe, O. Sigmund, Archive of Applied Mechanics, 1999, 69, 9-10.

[18] M. Y. Wang, X. M. Wang, D. M. Guo, Computer Methods in Applied Mechanics and Engineering, 2003, 192, 1-2.

[19] X. D. Huang, Y. M. Xie, John Wiley \& Sons, 2010.

[20] H. M. Zong, H. Y. Zhang, Y. Q. Wang, M. Y. Wang, J. Y. H. Fuh, Materials \& Design, 2018. 159.

[21] H. K. Zhang, Y. J. Luo, Z. Kang, Composite Structures, 2018, 195.

[22] F. W. Wang, Journal of the Mechanics and Physics of Solids, 2018, 114.

[23] D. S. Chen, M. Skouras, B. Zhu, W. Matusik, Science Advances, 2018, 4, 1.

[24] Z. L. Zhao, S. W, Zhou, X. Q. Feng, Y. M. Xie, Journal of the Mechanics and Physics of Solids, 2018, 119.

[25] H. X. Liu, Y. M. Hu, B. Zhu, W. Matusik, E. Sifakis, Acm Transactions on Graphics, 2018, 37,6 .

[26] Y. B. Deng, D. Mager, Y. Bai, T. Zhou, Z. Y. Liu, L. P. Wen, Y. H. Wu, J. G. Korvink, Computer Methods in Applied Mechanics and Engineering, 2018, 341, 1.

[27] N. Aage, E. Andreassen, B. S. Lazarov, O. Sigmund, Nature, 2017, 550, 7674. 
[28] Z. Lin, B. Groever, F. Capasso, A.W. Rodriguez, M. Loncar, Physical Review Applied, 2018, $9,4$.

[29] F. Callewaert, V. Velev, P. Kumar, A. V. Sahakian, K. Aydin, Scientific Reports, $2018,8$.

[30] F. Meng, B. H. Jia, X. D Huang, Advanced theory and simulations, 2018, 1, 12.

[31] W. B. Li, F. Meng, Y. F Chen, Y. F. Li, X. D. Huang, Advanced theory and simulations, 2019, 2,17 .

[32] J. K. Liu, A. T. Gaynor, S. K. Chen, Z. Kang, K. Suresh, A. Takezawa, L. Li, J. Kato, J.Y. Tang, C. C. L. Wang, L. Cheng, X. Liang, A. C. To, Structural and Multidisciplinary Optimization, 2018, 57, 6 .

[33] O. Sigmund, Mechanics of Materials, 1995, 20, 4.

[34] C. G. Mendez, J. M. Podesta, O. Lloberas-Valls, S. Toro, A. E. Huespe, J. Oliver, International Journal for Numerical Methods in Engineering, 2017, 112, 10.

[35] J. M. Podesta, C. M. Mendez, S. Toro, A. E. Huespe, Journal of the Mechanics and Physics of Solids, 2019, 128.

[36] Z. Kang, Y. Q. Wang, Computer Methods in Applied Mechanics and Engineering, 2011, 200, 49-52.

[37] X. D. Huang, A. Radman, Y. M. Xie, Computational Materials Science, 2011, 50, 6. 